\title{
Avaliação da qualidade da Atenção Primária à Saúde em Fortaleza, Brasil, na perspectiva dos usuários adultos no ano de 2019
}

\author{
Assessment of the quality of Primary Health Care in Fortaleza, \\ Brazil, from the perspective of adult service users in 2019
}

Lourrany Borges Costa (https://orcid.org/0000-0002-6334-8624) ${ }^{1,2}$

Maria Vaudelice Mota (https://orcid.org/0000-0002-4486-1895) ${ }^{1}$

Magda Moura de Almeida Porto (https://orcid.org/0000-0002-4806-2345) ${ }^{1}$

Carla Salles Gazeta Vieira Fernandes (https://orcid.org/0000-0002-1643-8643) ${ }^{2}$

Eveline Torquato Santos (https://orcid.org/0000-0001-5542-3420) ${ }^{2}$

João Pedro Marques de Oliveira (https://orcid.org/0000-0003-1819-3851) ${ }^{2}$

Thayná Custódio Mota (https://orcid.org/0000-0003-2466-8067) ${ }^{2}$

Andrea Lima da Silva Porto (https://orcid.org/0000-0002-5816-5233) ${ }^{1}$

Maria Nice Almeida Alencar (https://orcid.org/0000-0002-8079-7202) ${ }^{2}$

\footnotetext{
${ }^{1}$ Departamento de Saúde Comunitária, Faculdade de Medicina, Universidade Federal do Ceará (UFC). R. Prof. Costa Mendes 1281/1282, Rodolfo Teófilo. 60430-140 Fortaleza CE Brasil. lourranybc@unifor.br ${ }^{2}$ Centro de Ciências da Saúde, Faculdade de Medicina, Universidade de Fortaleza (UNIFOR). Fortaleza CE Brasil.
}

\begin{abstract}
Primary Health Care (PHC) is the "front door" and keystone of Brazil's public health system, meaning that the evaluation of the quality of primary care services is of utmost importance. Using the Primary Care Assessment Tool (PCAT), this study evaluated the performance of public PHC services in Fortaleza from the view of adult service users. We conducted a cross-sectional study of 233 adult service users from 19 primary care centers (PCCs) between June and December 2019, collecting data on the sociodemographic and epidemiological characteristics of users and structural features of PCCs. The association between user and PCC characteristics and primary care attribute scores was measured by multilevel logistic regression. Most participants were women, aged between 30 and 59 years, brown, house owners, had completed high school, did not own health insurance, and belonged to families with at least 4 members. The lowest and highest-scoring attributes were "first contact accessibility" and "first contact utilization" (2.8 and 8.1, respectively). The overall essential and general scores were 6.0 and 5.7, respectively. Having complete health teams, more community health workers, and a family and community medicine residency program had a positive effect on the general score $(p<0.05)$. Overall, the public services analyzed in Fortaleza received a low performance rating from the adult service users.
\end{abstract}

Key words Primary Health Care, Health Services Research, Quality of Health Care
Resumo A Atenção Primária à Saúde (APS) é porta de entrada e eixo estruturante do Sistema de Saúde brasileiro, o que impõe a necessidade de avaliação de sua qualidade. Este estudo objetivou avaliar os serviços públicos de Atenção Básica quanto aos atributos da APS por meio do PCATool na ótica de usuários em Fortaleza. Trata-se de um estudo transversal, de junho a dezembro de 2019, com 233 usuários adultos de 19 Unidades de APS (UAPS). Foram coletados dados sociodemográficos e epidemiológicos dos usuários e sobre a estrutura das UAPS. Utilizou-se modelo multinível para analisar características de usuários e UAPS em relação ao escore de APS medido via PCATool. Participantes foram, na maioria, mulheres, de 30 a 59 anos, pardos, com Ensino Médio completo, sem seguro privado, com moradia própria do tipo casa, de famílias até 4 pessoas. "Acessibilidade" obteve o menor escore, 2,8, e "Utilização" obteve o maior escore, 8,1. O Escore Essencial da APS foi 6,0, enquanto o Escore Geral foi 5,7. Houve influência positiva $(p<0,05)$ no Escore Geral por: equipes completas, maior número de agentes comunitários e presença de residência de Medicina de Família e Comunidade. Concluiuse que os serviços públicos de Fortaleza avaliados possuem baixo desempenho de APS na ótica dos usuários adultos.

Palavras-chave Atenção Primária à Saúde, Avaliação dos serviços, Qualidade da Assistência à Saúde 


\section{Introdução}

Vários eventos históricos marcam a expansão da Atenção Primária à Saúde (APS) no Brasil, desde a instituição do Sistema Único de Saúde (SUS), a criação do Programa Saúde da Família (PSF) e da Estratégia Saúde da Família (ESF), até a publicação da nova Política Nacional de Atenção Básica ${ }^{1}$. Em 2018, comemorou-se 30 anos do SUS, e apesar das grandes melhorias alcançadas, diversos desafios impedem sua efetivação e o fortalecimento da APS $^{2-5}$. Diversas mudanças ocorreram na APS brasileira em $2019^{6}$, abrangendo um novo modelo de monitoramento, avaliação e financiamento ${ }^{7}$.

Durante os anos, muitas iniciativas voltadas para avaliação e aperfeiçoamento da APS no país foram desenvolvidas. Um exemplo é o Programa Nacional de Melhoria do Acesso e da Qualidade da Atenção Básica (PMAQ-AB), lançado em 2011 pelo Ministério da Saúde (MS) ${ }^{8}$. Observa-se, porém, que as pesquisas de avaliação esbarram em dificuldades desde a falta de consensos sobre definições e dimensões de análise até a construção de indicadores, padronização de instrumentos e comparação dos resultados 9 .

Barbara Starfield ${ }^{10}$ sistematizou quatro atributos essenciais (acesso de primeiro contato, longitudinalidade, coordenação e integralidade) e três derivados (orientação familiar, orientação comunitária e competência cultural) da APS. A pesquisadora e colegas desenvolveram o PCATool (Primary Care Assessment Tool) ${ }^{10}$, instrumento para medição da presença e da extensão dos atributos da APS. Ele foi adaptado e validado para o Brasil em 2006 e lançado em 2010 como manual pelo $\mathrm{MS}^{11}$. Atualmente, é estimulado como ferramenta a ser aplicada em todo o país ${ }^{12}$. Revisões têm mostrado seu amplo uso nacional e internacional ${ }^{13,14} \mathrm{e}$, dentre os vários instrumentos para avaliar a APS, o PCATool é o mais capaz de fornecer subsídios para a qualificação da ESF ${ }^{15}$. Uma revisão sistemática analisou os estudos em âmbito mundial, de 2007 a 2015, que utilizaram o PCATool e observou-se que $68,2 \%(n=15)$ dos trabalhos selecionados eram brasileiros ${ }^{16}$. Um estudo realizado em Curitiba avaliou a concordância entre o PMAQ-AB e o PCATool e revelou não ser possível a utilização os padrões do PMAQ-AB como forma de mensurar todos os atributos da APS definidos por Starfield ${ }^{17}$.

Em Fortaleza-CE, a implantação do PSF teve início em 1997. Em 2006, foi realizado um concurso para formação de 460 equipes de Saúde da Família (eSF) além da criação da residência em Medicina de Família e Comunidade $(\mathrm{MFC})^{18}$. A organização da Rede de Atenção à Saúde (RAS) iniciou-se em 2013 com o "Modelo de Atenção às Condições Crônicas"19 e divisão em seis Secretarias Regionais (SER). Em 2019, Fortaleza possuía população estimada de 2.669.342 habitantes ${ }^{20}$. A Atenção Básica possuía 113 unidades de APS (UAPS), com cobertura aproximada de 61,45\% (1.624.290 pessoas), quando somadas eSF e equipes tradicionais $(\mathrm{eAB})^{21}$.

Ao longo dos anos, vários estudos avaliaram a qualidade da APS no Ceará, porém, a maioria utilizou questionários não validados ou métodos qualitativos. Até este momento, foram realizados apenas três estudos em Fortaleza utilizando o PCATool, com a versão para profissionais e a versão para criança ${ }^{22-25}$. Este trabalho tem como objetivo principal avaliar a qualidade dos serviços públicos de Atenção Básica em Fortaleza na perspectiva dos usuários adultos quanto aos atributos da APS, e analisar suas possíveis relações com características sociodemográficas e epidemiológicas de usuários e estruturais das unidades de saúde.

\section{Metodologia}

Trata-se de um estudo transversal, quantitativo, realizado de junho a dezembro de 2019. Foram selecionadas, por amostra aleatória estratificada, 36 UAPS de Fortaleza. A amostra de 463 participantes foi calculada com base no total de residentes em áreas cobertas por ESF na época de elaboração do projeto de pesquisa $(n=1.206 .983)$. Foi utilizada a fórmula para populações finitas, estimando como desfecho proporção hipotética de usuários que atribuiriam alto escore $(\geq 6,6)$ de APS para $50 \%$ das UAPS avaliadas, intervalo de confiança de $95 \%$, erro de $5 \%$, e fator de correção do efeito de desenho de 1,2. Por dificuldade de deslocamento e tempo para a realização da pesquisa, foram entrevistados apenas 233 indivíduos em 19 UAPS, em cinco das seis SER.

A amostragem dos participantes foi por conveniência. Foram entrevistados usuários maiores de 18 anos cadastrados nas UAPS selecionadas, que buscaram consulta médica ou com enfermeiro no dia da entrevista e que tinham se consultado com o médico ou enfermeiro na mesma unidade no mínimo duas vezes no último ano (considerando a consulta no dia da entrevista). Foram excluídos indivíduos que identificavam como serviço de referência um serviço fora de Fortaleza e moradores há menos de seis meses na área de adscrição da UAPS. 
A coleta de dados foi realizada via smartphones pelo pesquisador principal e por entrevistadores treinados seguindo as recomendações do manual do $\mathrm{MS}^{12}$. Deu-se pela aplicação de três instrumentos: questionário estruturado com variáveis sociodemográficas (raça/cor, idade, sexo, escolaridade, se possui plano de saúde privado, situação de moradia, tipo de domicílio, e número de moradores no domicílio) e epidemiológicas (se está gestante, está fumante, uso de álcool, uso de outras drogas, se tem pressão alta, diabetes, internação nos últimos 12 meses, diagnóstico de transtorno mental) dos usuários, baseado nas fichas de cadastro utilizadas pelos agentes comunitários de saúde (ACS); PCATool Brasil adulto; e questionário sobre características das unidades (população adscrita, número e composição das equipes, tempo para marcação de consultas, oferta de consultas, serviços disponíveis, presença de programas de residência), elaborado pelo autor e baseado em estudos prévios ${ }^{26,27}$, respondido pelos coordenadores de cada UAPS.

O PCATool contém 87 itens divididos em dez componentes relacionados aos atributos da APS: "grau de afiliação" (A), "acesso de primeiro contato - utilização" (B), "acesso de primeiro contato - acessibilidade" (C), "longitudinalidade" (D), "coordenação - integração de cuidados" (E), "coordenação - sistema de informações" (F), "integralidade - serviços disponíveis" (G), "integralidade - serviços prestados" (H), "orientação familiar" (I) e "orientação comunitária" (J). As respostas são em escala Likert ( $4=$ "com certeza, $\operatorname{sim}$ ", $3=$ "provavelmente sim", $2=$ "provavelmente não", $1=$ "com certeza, não" e 9 para "não sei/não lembro"). Os escores para cada atributo são calculados pela média das respostas dos itens que os compõem. O Escore Essencial (EE) é medido pela soma do escore médio dos atributos essenciais, dividido pelo número de componentes. $\mathrm{O}$ Escore Geral (EG) é medido pela soma do escore dos atributos essenciais e derivados, dividido pelo número de componentes. Para comparação da presença e extensão dos atributos, utilizou-se como ponto de corte valores de escores $\geq 6,6$, definidos como elevados ${ }^{28}$.

Os dados foram analisados com o software SPSS versão $24^{\circledR}$ (SPSS Inc., Chicago, Estados Unidos). Foi realizada análise descritiva de variáveis quantitativas independentes (escores PCATool para cada usuário entrevistado, escores médios de cada UAPS e para Fortaleza) e variáveis categóricas independentes (características dos usuários e características das UAPS). Para as comparações, utilizou-se o teste de Kruskal
-Wallis. Aplicou-se modelagem de regressão linear multinível para analisar os fatores preditivos, características dos indivíduos (nível 1) e das unidades (nível 2), que são capazes de explicar o EE e EG. Os resultados foram apresentados pelos coeficientes beta, seus respectivos intervalos de confiança e p-valor. Para cada variável inserida foi calculado a estatística t (teste Wald) para cálculo de variância e erro padrão. Adotou-se 5\% como nível de significância. Verificou-se a adequação do modelo utilizando análise de resíduos e a presença de colinearidade entre as variáveis ${ }^{29}$.

A pesquisa foi aprovada pelo Comitê de Ética da Pesquisa da Universidade Federal do Ceará.

\section{Resultados}

A maioria dos participantes foi mulheres (83,7\%, $\mathrm{n}=195)$, de 30 a 59 anos $(55,3 \%, \mathrm{n}=126)$, parda $(69,5 \%, n=162)$, com Ensino Médio completo $(39,5 \%, n=92)$, sem plano de saúde privado $(89,3 \%, \mathrm{n}=208)$, detentor de casa própria $(68,7 \%, \mathrm{n}=160)$ e pertencente a famílias de até quatro integrantes $(88 \%, n=205)$. Quanto às características epidemiológicas, $31,3 \%(n=73)$ dos participantes eram hipertensos, $12,9 \% \quad(n=30)$ diabéticos e $15,5 \% \quad(n=26)$ possuíam algum diagnóstico de transtorno mental. Uma minoria dos entrevistados estava gestante $(10,7 \%, n=25)$, $7,7 \%(n=18)$ era fumante e $14,2 \%(n=33)$ fazia uso de álcool (Tabela 1).

Sobre as 19 UAPS incluídas na pesquisa, 9 possuíam médicos de Família e Comunidade ( $\mathrm{n}=16$ de total de 77 médicos) compondo suas equipes, 6 unidades possuíam o programa de residência em MFC e 3 unidades o programa de residência multiprofissional em Saúde da Família. O número médio de dias para o agendar uma consulta eletiva (não pertencente a programa específico) foi de 49,7 dias.

A Tabela 2 apresenta a descrição dos atributos da APS avaliados em Fortaleza segundo os usuários adultos. O componente "acesso de primeiro contato - acessibilidade" foi o que apresentou menor escore médio, 2,8. O EE médio de Fortaleza foi 6,0, enquanto o EG médio foi 5,7.

A Tabela 3 mostra a comparação do resultado médio dos atributos medidos pelo PCATool em relação às características dos usuários. A maior idade foi associada a um escore mais elevado de "acesso de primeiro contato - acessibilidade" $(\mathrm{p}=0,018)$ e a um escore menor de "orientação familiar" $(p=0,004)$. Frequentar escola foi associado a maior "grau de afiliação" $(\mathrm{p}=0,046)$, 
Tabela 1. Características sociodemográficas e epidemiológicas dos entrevistados utilizando PCATool versão usuários adultos, Fortaleza, Brasil, 2019.

\begin{tabular}{|c|c|c|c|c|}
\hline \multirow{2}{*}{ Características sociodemográficas dos usuários } & & & \multicolumn{2}{|c|}{ Total $(\mathrm{N}=233)$} \\
\hline & & & $\mathbf{n}$ & $\%$ \\
\hline \multicolumn{5}{|l|}{ Raça/cor } \\
\hline Amarela & & & 4 & 1,7 \\
\hline Branca & & & 47 & 20,2 \\
\hline Parda & & & 162 & 69,5 \\
\hline Preta & & & 20 & 8,6 \\
\hline \multicolumn{5}{|l|}{ Sexo } \\
\hline Feminino & & & 195 & 83,7 \\
\hline Masculino & & & 38 & 16,3 \\
\hline \multicolumn{5}{|l|}{ Qual é o curso mais elevado que frequenta ou frequentou? } \\
\hline Nenhum & & & 5 & 2,1 \\
\hline Curso de Alfabetização & & & 11 & 4,7 \\
\hline Ensino Fundamental 1 a 4 séries & & & 43 & 18,5 \\
\hline Ensino Fundamental 5 a 8 séries & & & 37 & 15,9 \\
\hline Ensino Fundamental completo & & & 22 & 9,4 \\
\hline Ensino Médio completo & & & 92 & 39,5 \\
\hline Superior, Pós-graduação & & & 23 & 9,9 \\
\hline \multicolumn{5}{|l|}{ Situação de moradia } \\
\hline Alugado & & & 61 & 26,2 \\
\hline Cedido & & & 10 & 4,3 \\
\hline Financiado & & & 1 & 0,4 \\
\hline Ocupação & & & 1 & 0,4 \\
\hline Própria & & & 160 & 68,7 \\
\hline \multicolumn{5}{|l|}{ Tipo de Domicílio } \\
\hline Apartamento & & & 27 & 11,6 \\
\hline Casa & & & 202 & 86,7 \\
\hline Cômodo & & & 3 & 1,3 \\
\hline Outro & & & 1 & 0,4 \\
\hline \multicolumn{5}{|l|}{ No de moradores } \\
\hline 1 & & & 13 & 5,6 \\
\hline 2 & & & 41 & 17,6 \\
\hline 3 & & & 59 & 25,3 \\
\hline 4 & & & 62 & 26,5 \\
\hline$>4$ & & & 56 & 25 \\
\hline \multicolumn{5}{|l|}{ Possui plano de saúde privado? } \\
\hline Não & & & 208 & 89,3 \\
\hline Sim & & & 25 & 10,7 \\
\hline \multirow{2}{*}{ Características epidemiológicas dos usuários } & \multicolumn{2}{|c|}{ Não } & \multicolumn{2}{|c|}{ Sim } \\
\hline & $\mathbf{n}$ & $\%$ & $\mathbf{n}$ & $\%$ \\
\hline Está gestante? & 208 & 89,3 & 25 & 10,7 \\
\hline Está fumante? & 215 & 92,3 & 18 & 7,7 \\
\hline Faz uso de álcool? & 200 & 85,8 & 33 & 14,2 \\
\hline Faz uso de outras drogas? & 229 & 98,3 & 4 & 1,7 \\
\hline Tem pressão alta? & 160 & 68,7 & 73 & 31,3 \\
\hline Tem diabetes? & 203 & 87,1 & 30 & 12,9 \\
\hline Teve alguma internação nos últimos 12 meses? & 206 & 88,4 & 27 & 11,6 \\
\hline $\begin{array}{l}\text { Teve diagnóstico de transtorno mental por profissional } \\
\text { de saúde? }\end{array}$ & 197 & 84,5 & 36 & 15,5 \\
\hline
\end{tabular}

Fonte: Autoria própria. 
Tabela 2. Resumo descritivo dos escores globais e por atributo do questionário PCATool versão usuários adultos, Fortaleza, Brasil, 2019.

\begin{tabular}{lrrrrc}
\hline \multicolumn{1}{c}{ Escores } & Média & Mínimo & Máximo & $\begin{array}{c}\text { Desvio- } \\
\text { Padrão }\end{array}$ & $\begin{array}{c}\text { IC de } \\
\mathbf{9 5 \%}\end{array}$ \\
\hline Escore Essencial & 6 & 2,4 & 8,6 & 1,1 & $5,9-6,2$ \\
Escore Geral & 5,7 & 2,1 & 8,7 & 1,2 & $5,5-5,9$ \\
Por atributo & & & & & \\
$\quad$ Grau de Afiliação (A) & 9,7 & 6,7 & 10 & 0,9 & $9,6-9,9$ \\
Acesso de Primeiro Contato - Utilização (B) & 8,06 & 0 & 10 & 2,5 & $7,7-8,4$ \\
Acesso de Primeiro Contato - Acessibilidade (C) & 2,8 & 0 & 6,9 & 1,5 & $2,6-3,0$ \\
Longitudinalidade (D) & 6,4 & 0,7 & 10 & 1,9 & $6,1-6,6$ \\
Coordenação - Integração de Cuidados (E) & 7,01 & 0 & 10 & 2,8 & $6,3-7,7$ \\
Coordenação - Sistema de Informações (F) & 5,9 & 0 & 10 & 2,5 & $5,5-6,2$ \\
Serviços Disponíveis (G) & 5,3 & 2,1 & 9,1 & 1,3 & $5,1-5,5$ \\
Serviços Prestados (H) & 3,6 & 0 & 10 & 2,2 & $3,3-3,9$ \\
Orientação Familiar (I) & 4,9 & 0 & 10 & 3,1 & $4,5-5,3$ \\
Orientação Comunitária (J) & 4,02 & 0 & 10 & 2,3 & $3,7-4,3$ \\
\hline
\end{tabular}

Fonte: Autoria própria.

Tabela 3. Escore médio dos atributos pertencentes ao PCATool em relação as características sociodemográficas e epidemiológicas dos usuários, Fortaleza, Brasil, 2019.

\begin{tabular}{|c|c|c|c|c|c|c|c|c|c|c|}
\hline \multirow{2}{*}{ Característica do usuário } & \multicolumn{10}{|c|}{ Componente de Atributos } \\
\hline & $\mathbf{A}$ & B & $\mathrm{C}$ & $\mathbf{D}$ & E & $\mathbf{F}$ & G & $\mathbf{H}$ & I & $\mathbf{J}$ \\
\hline \multicolumn{11}{|l|}{ Raça/Cor } \\
\hline Outros & 9,8 & 7,5 & 2,5 & 6 & 6,1 & 5,9 & 5,1 & 3,4 & 4,8 & 3,7 \\
\hline Parda & 9,7 & 8,3 & 3 & 6,6 & 7,3 & 5,9 & 5,3 & 3,7 & 4,9 & 4,2 \\
\hline P-valor ${ }^{*}$ & 0,601 & 0,08 & 0,018 & 0,028 & 0,092 & 0,915 & 0,494 & 0,31 & 0,924 & 0,148 \\
\hline \multicolumn{11}{|l|}{ Frequenta escola? } \\
\hline Não & 9,7 & 8,4 & 2,8 & 6,6 & 7,2 & 6 & 5,2 & 3,8 & 5 & 4,1 \\
\hline Sim & 9,9 & 7,1 & 3 & 5,9 & 6,5 & 5,3 & 5,4 & 3,1 & 4,5 & 3,7 \\
\hline P-valor ${ }^{*}$ & 0,046 & 0 & 0,22 & 0,032 & 0,269 & 0,119 & 0,639 & 0,026 & 0,244 & 0,341 \\
\hline \multicolumn{11}{|l|}{ Escolaridade } \\
\hline Fundamental & 9,9 & 8,4 & 2,8 & 6,4 & 6,9 & 5,9 & 5,3 & 3,6 & 4,7 & 4,1 \\
\hline Médio & 9,6 & 7,9 & 2,7 & 6,3 & 7 & 5,9 & 5,3 & 3,5 & 4,9 & 4 \\
\hline Superior & 9,7 & 6,7 & 3,3 & 6,6 & 7,5 & 5,8 & 5,2 & 3,6 & 5,8 & 3,7 \\
\hline P-valor ${ }^{*}$ & 0,111 & 0,004 & 0,236 & 0,86 & 0,988 & 0,988 & 0,773 & 0,911 & 0,29 & 0,704 \\
\hline \multicolumn{11}{|l|}{ Situação de moradia } \\
\hline Outros & 9,7 & 8,2 & 2,9 & 6,2 & 7,3 & 6 & 5,2 & 3,4 & 4,7 & 3,3 \\
\hline Própria & 9,8 & 8 & 2,8 & 6,5 & 6,9 & 5,8 & 5,3 & 3,7 & 5 & 4,3 \\
\hline P-valor* & 1 & 0,473 & 0,529 & 0,428 & 0,594 & 0,483 & 0,545 & 0,379 & 0,629 & 0,002 \\
\hline \multicolumn{11}{|l|}{ Está gestante? } \\
\hline Não & 9,8 & 8,1 & 2,8 & 6,4 & 7 & 5,9 & 5,3 & 3,6 & 4,9 & 4,2 \\
\hline Sim & 9,5 & 7,6 & 3 & 6,5 & 10 & 6,1 & 5,3 & 3,3 & 5,1 & 2,9 \\
\hline P-valor ${ }^{*}$ & 0,111 & 0,283 & 0,555 & 0,611 & 0,347 & 0,712 & 0,982 & 0,5 & 0,748 & 0,01 \\
\hline \multicolumn{11}{|l|}{ Tem pressão alta? } \\
\hline Não & 9,7 & 8,1 & 2,8 & 6,3 & 7 & 5,9 & 5,4 & 3,6 & 5,1 & 4 \\
\hline Sim & 9,8 & 8 & 2,9 & 6,5 & 7 & 5,9 & 4,9 & 3,5 & 4,5 & 4 \\
\hline P-valor ${ }^{*}$ & 0,797 & 0,788 & 0,788 & 0,582 & 0,971 & 0,942 & 0,039 & 0,93 & 0,17 & 0,686 \\
\hline \multicolumn{11}{|c|}{ Teve diagnóstico de transtorno mental por profissional de saúde? } \\
\hline Não & 9,7 & 8 & 2,9 & 6,4 & 7 & 5,9 & 5,4 & 3,6 & 4,9 & 4,1 \\
\hline $\operatorname{Sim}$ & 9,7 & 8,2 & 2,4 & 6,5 & 7,2 & 5,9 & 4,9 & 3,7 & 4,6 & 3,4 \\
\hline P-valor* & 1 & 0,801 & 0,066 & 0,762 & 0,991 & 0,844 & 0,005 & 0,817 & 0,555 & 0,055 \\
\hline
\end{tabular}

${ }^{*}$ P-valor: teste de Kruskal-Wallis.

Fonte: Autoria própria 
menor "acesso de primeiro contato - utilização" $(\mathrm{p}<0,001)$, menor "longitudinalidade" $(\mathrm{p}=0,032)$ e menor "orientação familiar" $(p=0,026)$. A escolaridade teve influência no atributo "acesso de primeiro contato - utilização" ( $\mathrm{p}=0,004)$, com valores inversamente proporcionais ao número de anos de estudo.

Na Tabela 4, encontra-se o resultado do modelo linear multinível envolvendo características das UAPS (número total de eAB, número de médicos em eSF, número de ACS, tempo de espera e presença de residência de MFC) sobre o EG e o EE de Fortaleza. A variável "número total de eAB" causou impacto negativo no EG (diminuição de 0,298; p=0,007; IC95\% $-0,5--0,1)$ e no EE (diminuição de 0,272; $\mathrm{p}=0,013$; IC95\% $-0,5$ - -0,10). "Número de ACS" foi associado a um alto EG (aumento de 0,034; $\mathrm{p}=0,020$; IC95\% $0,0-0,1)$. A característica da UAPS "possui residência de MFC" teve impacto positivo no EG (aumento de 0,635; p=0,005; IC95\% 0,2 - 1,1) e no EE (aumento de 0,527; $\mathrm{p}=0,018$; IC95\% 0,1 1,0). Características dos usuários não foram preditores significativos para explicar o valor do EG ou EE no modelo multinível.

\section{Discussão}

As características sociodemográficas e epidemiológicas dos 233 participantes da pesquisa são corroboradas por vários estudos nacionais que mostram que, historicamente, dentre os usuários do SUS, há predomínio de mulheres, pretos e pardos, baixa escolaridade, baixa renda e sem posse de plano de saúde privado ${ }^{30-33}$. Não houve grande prevalência de pessoas com doenças crônicas, apesar de sabido que usuários com doenças crônicas utilizam mais os serviços de saúde ${ }^{34}$.

Quanto aos resultados do PCATool, ambos, EE e EG, ficaram abaixo de 6,6. Esses resultados demonstram a fraca inclinação dos serviços públicos de Atenção Básica de Fortaleza em relação aos atributos da APS na perspectiva dos usuários. Percebe-se, como detalhado a seguir, que os principais problemas que levaram aos baixos escores foram: baixa acessibilidade para queixas agudas e consultas de demanda espontânea, falta de acesso em horários noturnos e fins de semana, pouco acesso a informações por vias não presenciais, burocracia e longo tempo para agendamento de consultas, rigidez de agendas, baixo vínculo longitudinal entre usuários e equipes de saúde, falta de abordagem integral dos indivíduos durante a assistência, cuidado pouco centrado na pessoa, falhas na comunicação informacional e inte-

Tabela 4. Resultado do modelo de regressão multinível do escore geral e escore essencial segundo questionário PCATool versão usuários adultos de qualidade através das características individuais e da unidade de saúde, Fortaleza, Brasil, 2019.

\begin{tabular}{|c|c|c|c|c|c|}
\hline \multicolumn{6}{|c|}{ Escore Geral } \\
\hline Variável & Coef. $\beta$ & Erro padrão & Estatística t & P-valor & IC95\% \\
\hline Constante & 5,424 & 0,546 & 9,936 & $<0,001$ & $4,3-6,5$ \\
\hline Número total de eAB & $-0,298$ & 0,086 & $-3,457$ & 0,007 & $-0,4$ \\
\hline Número de médicos em ESF & 0,206 & 0,116 & 1,782 & 0,098 & $0,0-0,5$ \\
\hline Número de agentes comunitários & 0,034 & 0,013 & 2,579 & 0,02 & $0,0-0,1$ \\
\hline Tempo de espera (em dias) & $-0,001$ & 0,004 & $-0,273$ & 0,787 & $0,0-0,0$ \\
\hline Possui residência de MFC & 0,635 & 0,203 & 3,12 & 0,005 & $0,2-1,1$ \\
\hline \multicolumn{6}{|c|}{ Escore Essencial } \\
\hline Variável & Coef. $\beta$ & Erro padrão & Estatística t & P-valor & IC95\% \\
\hline Constante & 5,412 & 0,424 & 12,762 & $<0,001$ & $4,6-6,3$ \\
\hline Número total de $\mathrm{e} A \mathrm{~B}$ & $-0,272$ & 0,091 & $-2,975$ & 0,013 & $-0,4$ \\
\hline Número de médicos em ESF & 0,229 & 0,12 & 1,906 & 0,079 & $0,0-0,5$ \\
\hline Número de agentes comunitários & 0,027 & 0,014 & 2,009 & 0,062 & $0,0-0,1$ \\
\hline Tempo de espera (em dias) & $-0,001$ & 0,004 & $-0,238$ & 0,814 & $0,0-0,0$ \\
\hline Possui residência de MFC & 0,527 & 0,204 & 2,576 & 0,018 & $0,1-1,0$ \\
\hline
\end{tabular}


gração com outros níveis e redes de assistência, barreiras de acesso à atenção especializada, baixa oferta e diversidade de serviços resolutivos nas unidades de saúde, pequena inserção comunitária das ações das equipes de saúde, falta de orientação familiar e baixa participação dos usuários no controle social.

\section{Avaliação dos Atributos da Atenção Primária à Saúde}

A maioria dos entrevistados respondeu "com certeza, sim" para os itens de "acesso de primeiro contato - utilização", apontando a UAPS como fonte primária de atenção, entretanto, apenas $66,1 \%(n=154)$ buscam a UAPS quando tem um novo problema de saúde. Isso pode estar relacionado à concepção de que os serviços de APS são para atendimentos eletivos, pouco complexos ou de doenças crônicas, não casos agudos ou de urgência, levando-os a buscar Unidades de Pronto Atendimento $^{35}$. Dados nacionais de 2013 mostram que $74,4 \%$ dos brasileiros referiu possuir uma fonte usual de cuidados e que em mais de um terço dos casos era uma unidade de APS $^{36}$.

$\mathrm{O}$ atributo "acesso de primeiro contato acessibilidade", como em diversas pesquisas brasileiras, foi o atributo com pior desempenho ${ }^{14,37}$. Vários aspectos podem estar relacionados, desde despreparo dos profissionais até questões estruturais ou de organização de agenda, aumentando o tempo de espera e a insatisfação dos usuários $^{38,39}$. Ressalta-se que o horário padrão de funcionamento das UAPS é de 7 às 19 horas, de segunda à sexta-feira, limitando o acesso noturno e em fins de semana. Apenas 46,4\% ( $n=108)$ dos usuários responderam "com certeza, sim" sobre serem atendidos no mesmo dia que buscam a UAPS, alertando para a baixa acessibilidade a consultas não programadas ${ }^{40}$.

O atributo "longitudinalidade" revelou fraca relação interpessoal entre profissionais e usuários e a hegemonia de um cuidado centrado na doença. 62,2\% $(n=145)$ dos participantes responderam que mudariam de serviço, demonstrando insatisfação.

Quanto à "coordenação - integração de cuidados", a maioria respondeu que os profissionais de saúde se mostraram interessados sobre o agendamento e o atendimento em serviços especializados, semelhante a estudos prévios ${ }^{41,42}$. O escore médio de "coordenação - sistema de informações" foi de 5,9, apesar de todas as UAPS possuírem acesso à internet e prontuário eletrônico, além de fluxos de referência estabelecidos ${ }^{25}$.
O baixo valor de "integralidade - serviços disponíveis" em Fortaleza se assemelha a outros estudos nacionais ${ }^{16}$. Itens sobre vacinação, saúde bucal, prevenção de câncer do colo uterino e pré-natal apresentaram alto percentual de "com certeza, sim", variando entre $67,8 \%(n=158)$ a $95,3 \%(n=222)$. Tal achado evidencia a forte presença das ações programáticas referentes à saúde materno-infantil ${ }^{43-45}$. Em relação à "integralidade - serviços prestados”, os itens sobre alimentação, exercícios físicos e níveis de colesterol foram os que obtiveram maior percentual de "com certeza, sim", demonstrando que os profissionais tendem a valorizar aspectos preventivos de doenças crônicas $^{45}$. Outros dados são corroborados por vários trabalhos prévios nacionais, que mostram a baixa oferta na APS de procedimentos considerados cirúrgicos ou invasivos, como remoção de verrugas e suturas ${ }^{46,47}$ e aconselhamento sobre posse de armas de fogo e orientações sobre uso de cinto de segurança ou assentos para crianças ao andar de carro ${ }^{48}$.

"Orientação familiar" e "orientação comunitária” tiveram baixo desempenho. Destaca-se a alta proporção de "com certeza, não" sobre a realização de pesquisas de satisfação com usuários e pesquisas de identificação de problemas da comunidade. $65,2 \%$ responderam "com certeza, sim" sobre a realização de visitas domiciliares. Esse resultado deve-se, provavelmente, ao papel dos $\mathrm{ACS}^{49}$. Dados de 80 municípios de São Paulo mostram que $85 \%$ dos usuários da APS recebem visitas dos ACS, mas 58\% responderam que "nunca" ou "quase nunca" recebem de outros profissionais $^{50}$. Em Fortaleza, 85,8\% $\quad(n=200)$ dos participantes responderam "com certeza, não" sobre terem sido convidados a participar do Conselho local de Saúde, resultado semelhante ao encontrado em Juazeiro do Norte- $\mathrm{CE}^{51}$.

O Gráfico 1 compara os atributos da APS encontrados nos 4 estudos realizados em Fortaleza utilizando o PCATool entre os anos de 2013 e 2019. Interessante perceber que a maioria dos atributos é mais bem avaliada na opinião dos profissionais se comparada com os usuários, adulto ou criança, e que não houve grandes diferenças no valor dos atributos ao longo de 6 anos.

\section{Fatores que influenciam a avaliação da APS}

Os resultados apresentados na Tabela 3 corroboram estudos anteriores que mostraram que pessoas com maior escolaridade têm menor satisfação com os serviços de saúde ${ }^{52,53}$. Outros estudos, pelo contrário, mostraram associação 

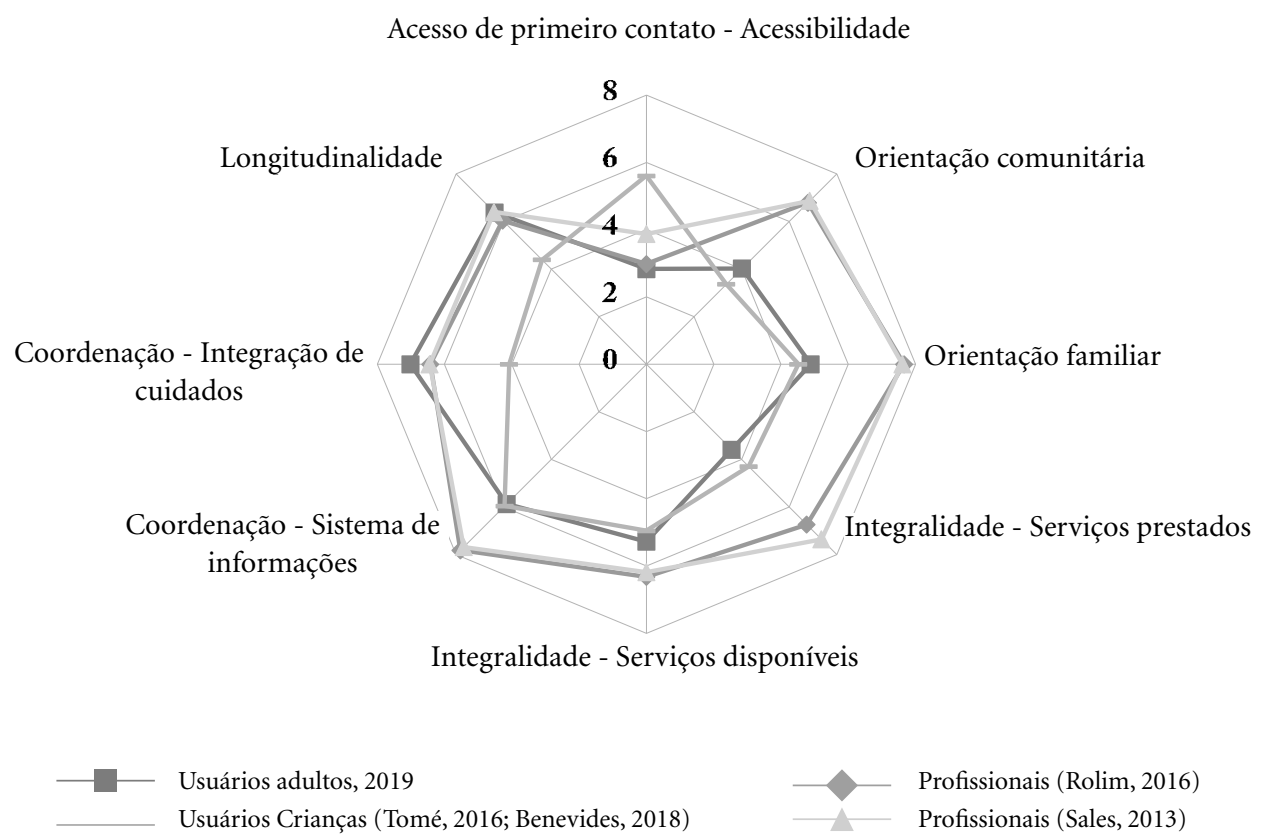

Profissionais (Rolim, 2016)

Profissionais (Sales, 2013)

Gráfico 1. Comparação entre os escores médios dos atributos da APS encontrados em 4 estudos realizados em Fortaleza-CE.

Fonte: Tomé ${ }^{22}$, Benevides et al. ${ }^{23}$, Sales ${ }^{24}$, Rolim$^{25}$.

positiva entre alta escolaridade e altos escores da APS ou alta satisfação. Porém, nestes trabalhos, esse fenômeno ocorreu com o aumento da idade. No estudo feito por Augusto et al. ${ }^{54}$, idosos com 80 anos ou mais e aqueles de maior escolaridade avaliaram melhor "acesso" e "longitudinalidade". A idade dos usuários também se mostrou um fator associado a altos escores de APS em um estudo realizado com homens em Teresina-PI. Quanto maior a idade dos participantes, melhor a avaliação de "longitudinalidade" e "sistema de informações" 55 .

$\mathrm{O}$ atributo "longitudinalidade" foi avaliado através do PCATool com 1.076 usuários adultos nos diferentes modelos assistenciais na APS de 32 municípios do Rio Grande do Sul em 2015. Foi então verificada a associação, por regressão, do atributo com características demográficas, socioeconômicas e de contexto de saúde (sexo, faixa etária, escolaridade, cor/etnia, situação conjugal, ter filhos, renda, emprego formal, plano de saúde privado, tipo de transporte utilizado para ir à unidade de saúde, região de saúde e modelo de APS). Mostraram-se associadas ao alto escore: ter idade de 60 anos ou mais e ser usuário do modelo de atenção com $\mathrm{ESF}^{56}$.
Entre os anos de 2003 e 2005, foram realizadas no Brasil duas pesquisas tendo como objetivo principal avaliar o desempenho do SUS. No ano de 2003, a pesquisa foi realizada em todo território nacional, chamada de Pesquisa Mundial de Saúde (PMS), com 3.932 usuários, e em 2005 foi realizada com amostra representativa nos estados do Rio de Janeiro e Pernambuco, conhecida como PMS com foco na Atenção Básica (PMS -AB), com 591 usuários. Os dados foram utilizados para identificar fatores associados ao grau de satisfação dos usuários. Aplicou-se modelo logístico multivariado, tendo como variável desfecho a satisfação e, como variáveis independentes sexo, idade, escolaridade, forma de pagamento de serviço, situação conjugal, autopercepção de saúde, doença de longa duração, depressão, tristeza, número de moradores por domicílio, tipo de construção, estratos geográficos e indicador de bens. Os resultados revelaram que ser jovem, usuário exclusivo do SUS, ter baixa escolaridade e autopercepção de saúde ruim gerou mais chances de insatisfação dos usuários com o SUS ${ }^{57}$.

No presente estudo, pessoas pardas atribuíram escores mais elevados aos atributos "acesso de primeiro contato - acessibilidade" e "longitu- 
dinalidade" quando comparados a pessoas de outras raças. Ressalta-se, porém, o alto percentual de pardos na pesquisa (69.5\%), o que pode ter influenciado o resultado. Salienta-se também que pessoas pardas utilizem mais os serviços públicos de $\mathrm{APS}^{30}$. Um estudo transversal em Belo Horizonte-MG, com idosos, mostrou uma melhor percepção da "longitudinalidade" por idosos de cor de pele não branca ${ }^{54}$. Esses resultados sugerem que os serviços de saúde avaliados contribuem para diminuição de iniquidades sociais.

Ter hipertensão ou ter o diagnóstico de um problema de saúde mental esteve associado a menor "integralidade - serviços disponíveis". Acredita-se que esses indivíduos com condições crônicas, por utilizarem mais a APS $^{58}$, são mais propensos a serem expostos a experiências ruins e, assim, emitir críticas ao cuidado recebido. No estudo feito por Augusto et al. ${ }^{54}$, os indivíduos hipertensos apresentaram pior percepção sobre a “coordenação do cuidado". Além disso, a existência de uma ou mais doenças crônicas, em comparação a nenhuma doença relatada, apresentou associação significativa e independente das condições sociodemográficas e do local de residência para pior percepção sobre "coordenação do cuidado", "orientação familiar" e "orientação comunitária" 54 . Influência no EG de maior idade e presença de uma ou mais doenças crônicas foram vistas em outros trabalhos ${ }^{59}$.

Ter moradia própria teve associação a um escore mais elevado de "Orientação Comunitária” ( $p=0,002)$. Esse resultado pode ser explicado pelo fato de que moradores antigos do território são capazes de avaliar com mais propriedade atividades do serviço de saúde que influenciam a comunidade, como as visitas de ACS. Estudos mostram que o tempo de moradia do usuário na área e a rotatividade dos profissionais também influenciam a "longitudinalidade"60-62. Além disso, municípios com mais de 100.000 habitantes e cobertura de ESF $<65 \%$, a exemplo de Fortaleza, possuem baixa "longitudinalidade" na visão de usuários ${ }^{56}$.

No nosso modelo multinível, características dos usuários não foram preditores significativos para explicar o valor do EG ou EE ${ }^{62}$. Porém, observou-se a influência positiva no EG dos fatores: eSF completas, maior número de ACS e presença de residência de MFC. Resultado semelhante foi encontrado na pesquisa realizada por Turci et al. ${ }^{63}$, em Belo Horizonte-MG. A pesquisa avaliou o desempenho da APS utilizando o PCATool com 538 enfermeiros e 147 gerentes da ESF, e analisou a influência das características estruturais e or- ganizacionais das unidades. Os fatores associados à melhor performance da APS $(\mathrm{p}<0,05)$ foram: disponibilidade de equipamentos/insumos, formação dos profissionais em Saúde da Família, presença do médico por mais de 30 horas semanais e quatro ou mais equipes por unidade ${ }^{63}$.

Corroborando a importância de eSF completas, uma revisão sistemática da literatura brasileira publicada entre os anos 2000 e 2013 buscou analisar a avaliação dos atributos da APS feita por usuários de unidades básicas de saúde, mediante utilização de adaptações do PCATool. Os achados mostraram que unidades com ESF são mais orientadas para à APS que as Unidades Básicas de Saúde tradicionais ${ }^{37}$.

Apesar de a PNAB não estipular um número mínimo de ACS por equipes, sabe-se da importância desde profissional, principalmente em relação à orientação familiar e comunitária da APS, facilitando os trabalhos de vigilância e de promoção da saúde realizados por equipe multiprofissional. Rodrigues et al. ${ }^{64}$ realizaram uma revisão integrativa, com 18 artigos, 16 brasileiros, de 2000 a 2011, sobre o papel da APS como coordenadora da RAS. Uma das potencialidades destacadas para o fortalecimento da APS foi o maior envolvimento do $\mathrm{ACS}^{64}$.

Assim como o presente estudo, outros trabalhos prévios também compararam os escores do PCATool em unidades de saúde que têm residência de MFC ou que têm profissionais com residência em Saúde da Família ou $\mathrm{MFC}^{64-66}$. Um estudo comparou o desempenho da APS medido pelo PCATool com usuários de unidades sem e com residência de MFC em Anápolis-GO. O EG na unidade sem residência foi de 4,5 e o EE foi 3,6 , enquanto na unidade com residência foram 6,8 e 5,5, respectivamente. Todos os atributos, exceto "coordenação do cuidado", obtiveram maior pontuação na unidade com residência. Além disso, um grupo focal com gestores mostrou que, embora inicialmente tenha ocorrido certa resistência ao trabalho dos residentes, houve significativa mudança no serviço, que passou a ser mais integral e resolutivo, resultando em satisfação da população e da própria equipe de profissionais ${ }^{65}$.

Leão e Caldeira ${ }^{66}$ verificaram a associação entre os atributos da APS e a qualificação profissional de médicos e enfermeiros em Montes Claros-MG, utilizando o PCATool versão criança. As equipes onde atuavam profissionais com residência em Saúde da Família ou residência de MFC apresentaram EG mais elevado do que as equipes onde os profissionais não possuíam tal qualificação $(\mathrm{p}=0,009)$. No modelo multivariado 
houve associação de alto escore de APS com profissional ter residência $(p<0,001)^{66}$.

Uma pesquisa realizada em Goiânia-GO, com 48 médicos e 44 enfermeiros, analisou o perfil de formação e qualificação dos profissionais da ESF com os escores da APS medidos pelo PCATool. Os resultados apontaram que os médicos apresentaram maior rotatividade no serviço e os enfermeiros maior experiência, qualificação profissional e vínculo com a ESF. A regressão logística indicou que a menor rotatividade de médicos e o aperfeiçoamento profissional associaram-se à melhor qualidade dos serviços ${ }^{67}$.

Outros estudos, que não utilizaram o $P C A$ Tool, também associam a presença de programas de residência a melhores indicadores de qualidade da APS. Herrera ${ }^{68}$ avaliou o impacto da residência em MFC na melhoria da APS em Gurupi-TO, de dezembro de 2017 a março de 2018, em 12 unidades de saúde. Participaram da pesquisa, 107 servidores de saúde (médicos, enfermeiros, técnicos de enfermagem e ACS), que responderam ao questionário QualiAB. Houve diferença significativa entre as unidades com e sem residência médica, sendo que os itens que mais contribuíram foram: "Educação em saúde" e "Recursos materiais, procedimentos e insumos básicos". Concluiu-se que o programa de residência promoveu melhoria na qualidade da APS municipal ${ }^{68}$.

Um estudo em Montes Claros-MG avaliou a influência da residência de MFC e da residência multiprofissional em Saúde da Família pelo monitoramento dos indicadores do PMAQ do $1^{\circ} \mathrm{e}$ $2^{\circ}$ ciclos (anos de 2011/2012 e 2013/2014) das equipes com residentes e ou egressos de residência. Fizeram parte desse estudo 17 equipes do $1^{\circ}$ e 26 equipes do $2^{\circ}$ ciclo. Foram utilizados como varáveis, indicadores de desempenho e padrões de qualidade das equipes. Observou-se melhora significativa do $1^{\circ}$ para o $2^{\circ}$ ciclo, considerando os indicadores "acesso à marcação de consulta" e "acolhimento a demanda espontânea". Quanto à satisfação do usuário, todas as equipes do $2^{\circ}$ ciclo foram avaliadas como acima da média ${ }^{69}$. Esses resultados sugerem que a residência de MFC pode auxiliar o acesso à APS, atributo mais mal avaliado em Fortaleza e em várias outras cidades brasileiras.

\section{Limitações do estudo}

São limitações o fato de ser um estudo transversal, sujeito às limitações próprias desse delineamento e o uso de amostragem por conveni- ência, que não possui validade externa, além da quantidade de participantes inferior ao calculado para a amostra. Também constitui limitação a entrevista dos usuários feita após atendimento devido o viés de cortesia. É importante reforçar que a análise da qualidade da APS de Fortaleza, neste trabalho, foi baseada apenas na ótica dos usuários participantes.

\section{Conclusão}

Os resultados da pesquisa mostram que, sob a ótica dos usuários participantes, em média, as UAPS selecionadas apresentaram baixa presença e extensão dos atributos da APS. Apesar das limitações do estudo e a impossibilidade de generalização dos resultados para todo o munícipio de Fortaleza, esses resultados podem guiar profissionais de saúde e gestores locais e nacionais na busca por estratégias de fortalecimento da APS.

Uma série de estratégias podem ser implementadas para melhorar a qualidade da APS em Fortaleza e, consequentemente, a satisfação dos usuários, como $^{70,71}$ : aumento do financiamento; ampliação do acesso, com adoção de outros modelos de agendamento, como acesso avançado, acesso não presencial com incorporação de ferramentas digitais para comunicação entre equipe de saúde e usuários, horário estendido e em fins de semana, além de melhor gestão de agendas; ofertar ações e serviços de saúde abrangentes, formulando uma carteira de serviços com garantia de recursos e de capacitação profissional para execução plena e eficiente da carteira; ampliação da atuação clínico-assistencial de todos os profissionais das equipes de saúde; expansão da informatização plena da rede assistencial; aperfeiçoamento do sistema de regulação; otimização da oferta de recursos humanos com formação qualificada; apoio assistencial às equipes de saúde; estímulo a participação dos usuários e dos atores sociais; e a avaliação contínua e sistemática dos serviços de saúde.

Recomendamos a valorização dos ACS, dada a importância da atuação desses profissionais para a organização e qualidade da APS, por serem articuladores entre as demandas da comunidade e as ações dos serviços de saúde. Preconiza-se o fortalecimento da residência médica em Medicina de Família e Comunidade como parte de um projeto político que valorize a APS. Além disso, sugere-se a utilização sistemática do PCATool como instrumento avaliativo dos serviços locais, pelo potencial de permitir o monitoramento das 
atividades que compõem cada atributo, possibilitando a comparação com outras regiões, nacionais e internacionais. É necessário reconhecer que apenas através de uma APS forte é que será alcançado um sistema de saúde universal sustentável capaz de oferecer serviços com qualidade, custo-efetivos, resolutivos, abrangentes e continuados, e que possa diminuir iniquidades e promover desenvolvimento e justiça social.

\section{Colaboradores}

LB Costa realizou a concepção, curadoria de dados, análise, investigação, metodologia, administração do projeto, redação, revisão e edição do artigo final. MV Mota e MMA Porto colaboraram com concepção, metodologia, administração do projeto, revisão de redação e edição do artigo final. CSGV Fernandes, ET Santos, JPM Oliveira, TC Mota, ALS Porto e MNA Alencar contribuíram com a investigação. 


\section{Referências}

1. Almeida ER, Sousa ANA, Brandão CC, Carvalho FFB, Tavares G, Silva KC. Política Nacional de Atenção Básica no Brasil: uma análise do processo de revisão (2015-2017). Rev Panam Salud Publica 2018; 42:1-8.

2. Bahia L. Trinta anos de Sistema Único de Saúde (SUS): uma transição necessária, mas insuficiente. Cad Saude Publica 2018; 34(7):e00067218.

3. Bousquat A, Giovanella L, Campos EMS, Almeida PF, Martins CL, Mota PHDS, Mendonça MHM, Medina MG, Viana ALD, Fausto MCR, Paula DB. Atenção primária à saúde e coordenação do cuidado nas regiões de saúde: perspectiva de gestores e usuários. Cien Saude Colet 2017; 22(4):1141-1154.

4. Giovanella L, Mendonça MHM, Buss PM, Fleury S, Gadelha CAG, Galvão LAC, Augusto CL, Santos RF. De Alma-Ata a Astana. Atenção primária à saúde e sistemas universais de saúde: compromisso indissociável e direito humano fundamental. Cad Saude Publica 2019; 35(3):e00012219.

5. Massuda A, Hone T, Leles FAG, Castro MC, Atun R. The Brazilian health system at crossroads: Progress, crisis and resilience. BMJ Glob Heal 2018; 3(4):1-8.

6. Harzheim E, Santos CMJ, D'Avila OP, Wollmann L, Pinto LF. Bases para a Reforma da Atenção Primária à Saúde no Brasil em 2019: mudanças estruturantes após 25 anos do Programa de Saúde da Família. Rev Bras Med Familia Comun 2020; 15(42):2354.

7. Sellera PEG, Pedebos LA, Harzheim E, de Medeiros OL, Ramos LG, Martins C, D’Ávila OP. Monitoramento e avaliação dos atributos da Atenção Primária à Saúde em nível nacional: novos desafios. Cien Saude Colet 2020; 25(4):1401-1412.

8. Gomes LB, Barbosa MG, Ferla AA. Atenção básica: olhares a partir do programa nacional de melhoria do acesso e da qualidade-(PMAQ-AB) [Internet]. Rede UNIDA; 2016 [acessado 2020 mar 21]. Disponível em: http://www.redeunida.org.br/editora/bibliotecadigital/serie-atencao-basica-e-educacao-na-saude/ atencao-basica-olhares-a-partir-do-programa-nacional-de-melhoria-do-acesso-e-da-qualidade2013-pmaq-ab-pdf/view.

9. Facchini LA, Tomasi E, Dilélio AS. Qualidade da Atenção Primária à Saúde no Brasil: avanços, desafios e perspectivas. Saude Debate 2018; 42(esp. 1):208-223.

10. Starfield B. Atenção Primária: Equilíbrio entre necessidades de saúde, serviços e tecnologia. Brasília: UNESCO, MS; 2002.

11. Shi L, Starfield B, Xu J. Validating the adult primary care assessment tool. J Fam Pract 2001; 50(2):161.

12. Harzheim E, Gonçalves MR, Oliveira MC, Agostinho MR, Hauser L. Manual do Instrumento de Avaliação da Atenção Primária à Saúde Primary Care Assessment Tool PCATool-Brasil [Internet]. 2010 [acessado 2017 out 6]. Disponível em: http://bvsms.saude.gov.br/bvs/ publicacoes/manual_avaliacao_pcatool_brasil.pdf.

13. Reis JG, Harzheim E, Nachif MCA, Freitas JC, D’Ávila O, Hauser L, Martins C, Pedebos LA, Pinto LF. Creation of the primary health care secretariat and its implications for SUS. Cien Saude Colet 2019; 24(9):34573462.
14. Prates ML, Machado JC, Silva LS, Avelar PS, Prates LL, Mendonça ET, Costa GD, Cotta RMM. Desempenho da Atenção Primária à Saúde segundo o instrumento PCATool: uma revisão sistemática. Cien Saude Colet 2017; 22(6):1881-1893.

15. Figueiredo AM, Kuchenbecker RS, Harzheim E, Vigo Á, Hauser L, Chomatas ERV. Análise de concordância entre instrumentos de avaliação da Atenção Primária à Saúde na cidade de Curitiba, Paraná, em 2008. Epidemiol Serv Saude 2013; 22(1):41-48.

16. D'Ávila OP, Pinto LFS, Hauser L, Gonçalves MR, Harzheim E. O uso do primary care assessment tool (PCAT): Uma revisão integrativa e proposta de atualização. Cien Saude Colet 2017; 22(3):855-865.

17. Fracolli LA, Gomes MFP, Nabao FRZ, Santos MS, Cappellini VK, Almeida ACC. Primary health care assessment tools: a literature review and metasynthesis. Cien Saude Colet 2014; 19(12):4851-4860.

18. Aguiar MTMR. Avaliação da implantação de Programa de Residência em Medicina de Família e Comunidade de larga escala em Capital do Nordeste [dissertação]. Fortaleza: Universidade Federal do Ceará; 2009.

19. Oliveira BN. Ações da Estratégia Saúde da Família na perspectiva das Redes de Atenção à Saúde: um estudo avaliativo [dissertação]. Fortaleza: Universidade Estadual do Ceará; 2015.

20. Instituto Brasileiro de Geografia Estatística (IBGE). Fortaleza Ceará [Internet]. [acessado 2020 ago 20]. Disponível em: https://cidades.ibge.gov.br/brasil/ce/ fortaleza/panorama.

21. Brasil. Ministério da Saúde (MS). e-gestor, cobertura da atenção básica [Internet]. 2019 [acessado $2020 \mathrm{fev}$ 20]. Disponível em: https://egestorab.saude.gov.br/ paginas/acessoPublico/relatorios/relHistoricoCoberturaAB.xhtml.

22. Tomé MABG. Orientação familiar e comunitária sob a ótica das internações por condições sensíveis em crianças de 0 até 5 anos Fortaleza 2016 [dissertação]. Fortaleza: Universidade Federal do Ceará; 2017.

23. Benevides JL, Gubert FA, Tomé MABG, Vasconcelos PF, Dodt RCM, Sales SRC. Primary care features in child hospitalization: First-contact access and Longitudinality. Rev Rede Enferm Nord 2018; 19:e3481.

24. Sales NC. Avaliação da qualidade em Atenção Primária em Saúde: comparação entre os instrumentos PCAToole competências essenciais de gerentes dos Centros de Saúde da Família de Fortaleza-CE [dissertação]. Fortaleza: Universidade Estadual do Ceará; 2013.

25. Rolim LB. Avaliação dos atributos da Atenção Primária à Saúde de Fortaleza-CE: estudo através do PCATool-Brasil, versão profissionais [dissertação]. Fortaleza: Fundação Oswaldo Cruz, Rede Nordeste de Formação em Saúde da Família; 2016.

26. Vitória AM. Avaliação da estrutura e dos processos na Atenção Primária à Saúde em Chapecó: um estudo de adequação [dissertação]. Porto Alegre: Universidade Federal do Rio Grande do Sul; 2012.

27. Brasil. Ministério da Saúde (MS). Departamento de Atenção Básica. Manual de Estrutura Física das Unidades Básicas de Saúde. Brasília: MS; 2006. 
28. Harzheim E, Goncalves MR, D'Avila OP, Hauser L Pinto LF. Estudos de PCATool no Brasil. In: De Mendonça MHM, Matta GC, Gondim R, Giovanella L. Atenção primária à saúde no Brasil: conceitos, práticas e pesquisa. Rio de Janeiro: Editora Fiocruz; 2018. p. 493-525.

29. Vidal TB, Rocha SA, Harzheim E, Hauser L, Tesser CD. Modelos de agendamento e qualidade da atenção primária: estudo transversal multinível. Rev Saude Publica 2019; 53(1):38-53.

30. Ribeiro MCSA, Barata RB, Almeida MF, Silva ZP. Perfil sociodemográfico e padrão de utilização de serviços de saúde para usuários e não-usuários do SUS PNAD 2003. Cien Saude Colet 2006; 11(4):1011-1022.

31. Dilélio AS, Tomasi E, Thumé E, Silveira DS, Siqueira FCV, Piccini RX, Silva SM, Nunes BP, Facchini LA. Patterns in the use of outpatient care in Brazil by patients treated through the Brazilian Unified National Health System, private health insurance, and out-of-pocket medical care. Cad Saude Publica 2014; 30(12):2594-2606

32. Guibu IA, Moraes JC, Guerra Junior AA, Costa EA, Acurcio FA, Costa KS, Karnikowski MGO, Soeiro OM, Leite SN, Álvares J. Características principais dos usuários dos serviços de atenção primária à saúde no Brasil. Rev Saúde Pública 2017; 51(Supl. 2):1s-13s.

33. Fontenelle LF, Sarti TD, Camargo MBJ, Maciel ELN, Barros AJD. Utilization of the Brazilian public health system by privately insured individuals: a literature review. Cien Saude Colet 2019; 35(4):e00004118.

34. Malta DC, Bernal RTI, Lima MG, Araújo SSC, Silva MMA, Freitas MIF, Barros MBA. Doenças crônicas não transmissíveis e a utilização de serviços de saúde: análise da Pesquisa Nacional de Saúde no Brasil. Rev Saude Publica 2017; 51(1):1-10.

35. Pimentel SK, Soares MC, Mazepa MM, Kato PVK, Lima RL, Soares MEC. Análise dos motivos para uso do serviço de urgência e emergência pelo paciente não grave. Rev Med UFPR 2016; 3(3):109-113.

36. Dourado I, Medina MG, Aquino R. The effect of the Family Health Strategy on usual source of care in Brazil: data from the 2013 National Health Survey (PNS 2013). Int J Equity Health 2016; 15(1):151

37. Paula WKAS, Samico IC, Caminha MFC, Batista Filho M, Silva SL. Avaliação da atenção básica à saúde sob a ótica dos usuários: uma revisão sistemática. Rev Esc Enferm USP 2016; 50(2):335-345.

38. Coutinho LRP, Barbieri AR, Santos MLM. Acolhimento na Atenção Primária à Saúde: revisão integrativa. Saude Debate 2015; 39(105):514-524.

39. Soares APG, Andrade JT. Cuidado humanizado na atenção primária à saúde: Demanda por serviços e atuação profissional na rede de atenção primária à saúde - Fortaleza, Ceará, Brasil. Rev Port Saude Publica 2014; 32(1):61-68.

40. Tesser CD, Norman AH, Vidal TB. Acesso ao cuidado na Atenção Primária à Saúde brasileira: situação, problemas e estratégias de superação. Saude Debate 2018; 42(esp. 1):361-378.

41. Souza GT, Alves BA, Tacla MTGM, Collet N, Toso BRGO. Avaliação do princípio da coordenação na atenção primária à saúde da criança em Londrina-PR. Semin Cirn Biol Saude 2015; 36(1):39-46.
42. Carneiro SMM, Silva MGC, Pinto FJM, Melo DMS Gomes JM. Avaliação do atributo coordenação da Atenção Primária à Saúde: aplicação do PCATool a profissionais e usuários. Saude Debate 2014; 38(esp.):279-295.

43. Araujo ACAF, Silva ANE, Ribeiro MGC, Rocha SS, Andrade EMLR, Nogueira LT. Avaliação da Atenção Primária à Saúde sob a ótica de cuidadores de crianças: revisão integrativa. Rev Esc Enferm USP 2019; 53:e03527.

44. Sala A, Luppi CG, Simões O, Marsiglia RG. Integralidade e atenção primária à saúde: 44 . Avaliação na perspectiva dos usuários de unidades de saúde do município de São Paulo. Saude Soc 2011; 20(4):948-960.

45. Pinto MEB. Promoção da saúde e atenção primária à saúde em Porto Alegre [tese]. Porto Alegre: Universidade Federal do Rio Grande do Sul; 2012.

46. Pucci VR. Avaliação do atributo da integralidade na atenção primária à saúde em municípios da região centro-oeste no Rio Grande do Sul: PCATool-Brasil versões profissionais [dissertação]. Santa Maria: Universidade Federal de Santa Maria; 2017.

47. Lima JG, Giovanella L, Fausto MCR, Bousquat A, Silva EV. Atributos essenciais da Atenção Primária à Saúde: resultados nacionais do PMAQ-AB. Saude Debate 2018; 42(esp. 1):52-66.

48. Araújo LYBVS. A Avaliação da Atenção Primária: um olhar preliminar através do PCATool em Manaus, Amazonas. Aps Em Rev 2019; 1(2):98-111.

49. Daschevi JM, Tacla MTGM, Alves AB, Toso BRGO, Collet N. Avaliação dos princípios da orientação familiar e comunitária da atenção primária à saúde da criança. Semin Cien Biol Saude 2015; 36(1):31-38.

50. Catanante GV, Hirooka LB, Porto HS, Bava CGGCM. Participação social na atenção primária à Saúde em direção à Agenda 2030. Cien Saude Colet 2017; 22(12):3965-3974.

51. Rodrigues EMD, Bispo GMB, Costa MS, Oliveira CAN, Freitas RWJR, Damasceno MMC. Avaliação do atributo "Orientação Comunitária" na óptica do usuário adulto da atenção primária. Rev Bras Enferm 2019; 72(3):632-639.

52. Brandão RBAL. Avaliação da atenção primária pela perspectiva dos usuários: adaptação do instrumento EUROPEP para grandes centros urbanos brasileiros uma aplicação na comunidade de Manguinhos [dissertação]. Rio de Janeiro: ENSP/Fiocruz; 2011.

53. Passero LG. Insatisfação do usuário da atenção básica com o SUS: análise multinível da pesquisa da ouvidoria [dissertação]. Porto Alegre: Universidade Federal do Rio Grande do Sul; 2013.

54. Augusto DK, Lima-Costa MF, Macinko J, Peixoto SV. Fatores associados à avaliação da qualidade da atenção primária à saúde por idosos residentes na Região Metropolitana de Belo Horizonte, Minas Gerais, 2010. Epidemiol Serv Saude Rev Sist Unico Saude Bras 2019; 28(1):e2018128.

55. Silva AN, Silva SA, Silva ARV, Araújo TME, Rebouças CBA, Nogueira LT. A avaliação da atenção primária a saúde na perspectiva da população masculina. Rev Bras Enferm 2018; 71(2):236-243. 
56. Kessler M, Lima SBS, Weiller TH, Lopes LFD, Ferraz L, Eberhardt TD, Soares RSA, Trindade LL. Longitudinalidade do cuidado na atenção primária: avaliação na perspectiva dos usuários. Acta Paul Enferm 2019; 32(2):186-193.

57. Gouveia GC, Souza WV, Luna CF, Souza-Júnior PRB, Szwarcwald CL. Satisfação dos usuários do sistema de saúde brasileiro: Fatores associados e diferenças regionais. Rev Bras Epidemiol 2009; 12(3):281-296.

58. Rzewuska M, Azevedo-Marques JM, Coxon D, Zanetti ML, Zanetti ACG, Franco LJ, Santos JLF. Epidemiology of multimorbidity within the Brazilian adult general population: Evidence from the 2013 National Health Survey (PNS 2013). PLoS One 2017; 12(2):1-13.

59. Rech MRA, Hauser L, Wollmann L, Roman R, Mengue SS, Kemper ES, Souza ARF, Alfaro, G, Tasca R, Harzheim E. Qualidade da atenção primária à saúde no Brasil e associação com o Programa Mais Médicos. Rev Panam Salud Publica 2018; 42:1-11.

60. Paula CC, Silva CB, Nazário EG, Ferreira T, Schimith $\mathrm{MD}$, Padoin SMM. Fatores que interferem no atributo longitudinalidade da atenção primária à saúde: revisão integrativa. Rev Eletr Enferm 2015; 17:4.

61. Baricati CCA. A longitudinalidade do cuidado na atenção básica à luz da experiência dos usuários com hipertensão arterial [tese]. Londrina: Universidade Estadual de Londrina; 2016.

62. Turci MA, Costa MFL, Macinko JA. Avaliação da Atenção Primária e a percepção do usuário sobre a qualidade da assistência: um estudo em cidade brasileira. Aps Em Rev 2019; 1(1):3-17.

63. Turci MA, Costa MFL, Macinko J. Influência de fatores estruturais e organizacionais no desempenho da atenção primária à saúde em Belo Horizonte, Minas Gerais, Brasil, na avaliação de gestores e enfermeiros. Cad Saude Publica 2015; 31(9):1941-1952.

64. Rodrigues LBB, Silva PC dos S, Peruhype RC, Palha PF, Popolin MP, Crispim J de A, Pinto IC, Monroe AA, Arcêncio RA. A atenção primária à saúde na coordenação das redes de atenção: Uma revisão integrativa. Cien Saude Colet 2014; 19(2):343-352.

65. Dutra ACF, Martins I, Leite ICS, Porto JM, Cintra PVDAC. Residência de Medicina de Família e Comunidade: avaliação da implementação do programa no município de Anápolis. In: Anais XII Mostra de Saúde [Internet]. [acessado 2020 fev 20]. Disponível em: http://periodicos.unievangelica.edu.br/index.php/ educacaoemsaude/article/view/2320/1981.
66. Leão CDA, Caldeira AP. Avaliação da associação entre qualificação de médicos e enfermeiros em atenção primária em saúde e qualidade da atenção. Cien Saude Colet 2011; 16(11):4415-4423.

67. Oliveira MPRD, Menezes IHCF, Sousa LM, Peixoto MRG. Formação e qualificação de profissionais de saúde: fatores associados à qualidade da atenção primária. Rev Bras Educ Med 2016; 40(4):547-559.

68. Herrera SDC. Residência de medicina da família e comunidade promove melhoria na atenção básica de saúde? [dissertação]. Palmas: Universidade Federal do Tocantins; 2018.

69. Gonçalves DP, Cardoso MCLR, Silva TLS, Magalhães TA, Araújo A. Análise do desempenho das Residências Médicas de Medicina de Família e Comunidade e Multiprofissional em Saúde da Família segundo os indicadores do PMAQ-AB das equipes da Atenção Primária. Rev Bras Med Familia Comun 2019; 14(41):1879.

70. Tasca R, Massuda A, Carvalho WM, Buchweitz C, Harzheim E. Recomendações para o fortalecimento da atenção primária à saúde no Brasil. Rev Panam Salud Publica 2020; 44:e4.

71. Rocha SA, Bocchi SCM, Godoy MF. Acesso aos cuidados primários de saúde: Revisão integrativa. Physis 2016; 26(1):87-111.

Artigo apresentado em 27/08/2020

Aprovado em 18/11/2020

Versão final apresentada em 20/11/2020

Editores-chefes: Maria Cecília de Souza Minayo, Romeu Gomes, Antônio Augusto Moura da Silva 